\title{
Datennutzung der VHS- und Verbundstatistik. Potenziale für Bildungsforschung, -politik und -praxis
}

\author{
Sarah Widany $\cdot$ Elisabeth Reichart $(\mathbb{D}) \cdot$ Ingrid Ambos $\cdot$ Markus Huff
}

Eingegangen: 18. Dezember 2018 / Angenommen: 16. April 2019 / Online publiziert: 20. Mai 2019

(C) Der/die Autor(en) 2019

Zusammenfassung Anbieterstatistiken sind eine wichtige Säule der fragmentierten Weiterbildungsstatistik. Der Beitrag erörtert die besonderen Voraussetzungen für die Entstehung und Nutzung der „Volkshochschulstatistik“ und der „Verbundstatistik“ als sogenannte freiwillige Anbieterstatistiken der öffentlich geförderten Weiterbildung, die in Zusammenarbeit von Weiterbildungsverbänden und dem Deutschen Institut für Erwachsenenbildung (DIE) erstellt werden. Wir beschreiben die Nutzung dieser Daten in den Kontexten von Bildungsforschung, Bildungspolitik und Bildungspraxis. Zudem wird das mit der Revision der Statistik neu erschlossene Nutzungspotenzial aufgezeigt.

Schlüsselwörter Weiterbildungsstatistik · Weiterbildungsanbieter ·

Bildungspraxis · Bildungspolitik · Bildungsforschung $\cdot$ Volkshochschule

\section{Usage of the data from statistics on adult education centers in Germany. Potentials for research, politics and practice}

Abstract Provider statistics are an important pillar in a fragmented structure of adult education statistics. The article discusses the special prerequisites for the emergence

\footnotetext{
Dr. S. Widany $\cdot$ Dr. E. Reichart $(\varangle) \cdot$ I. Ambos $\cdot$ PD Dr. M. Huff

Leibniz-Zentrum für Lebenslanges Lernen e. V., Deutsches Institut für Erwachsenenbildung, Bonn, Deutschland

E-Mail: reichart@die-bonn.de

Dr. S. Widany

E-Mail: widany@die-bonn.de

I. Ambos

E-Mail: reichart@die-bonn.de

PD Dr. M. Huff

E-Mail: huff@die-bonn.de
} 
and use of the German "Volkshochschulstatistik" [Statistics on Adult Education Centres in Germany] and the "Verbundstatistik" [Statistics on Continuing Education Network], which are compiled as voluntary statistics by means of cooperation between the adult education associations and the German Institute for Adult Education. We outline the use of these statistics in the contexts of educational research, educational policy and educational practice. In addition, the analytic potential which emerges from the revision of the statistics will be pointed out.

Keywords Provider statistics - Adult education providers - Educational research . Educational practice $\cdot$ Educational policy $\cdot$ Adult education centres

\section{Einleitung}

Die datengestützte Beobachtung des Weiterbildungssystems basiert auf einer Vielzahl von Datengrundlagen, mit denen jeweils Ausschnitte der Weiterbildungsrealität dargestellt werden können. Anbieterstatistiken, die Daten zur Struktur und Organisation von Weiterbildungseinrichtungen, zum Angebotsspektrum und zu Teilnahmefällen als realisierte Nachfrage des Angebots liefern, sind ein wichtiger Baustein in diesem Gerüst. Während vergleichbare Daten aus anderen Bildungsbereichen in öffentlicher Verantwortung auf der Grundlage landesweiter und länderübergreifender sowie bundesweiter beziehungsweise europäischer gesetzlicher Regelungen konzipiert, erhoben und bereitgestellt werden, basieren Anbieterstatistiken in der Weiterbildung auf der freiwilligen Selbstorganisation von Einrichtungen und deren Verbänden. Diese Organisationsform bringt besondere Bedingungen für die Datengenese mit sich. Der Regelungskontext besteht nicht aus amtlichen Vorschriften, sondern aus einem Interessenausgleich zwischen Akteuren aus drei interdependenten Nutzungskontexten: Forschung, Politik und Praxis.

Der Beitrag fragt nach den Voraussetzungen für die erfolgreiche Genese und Verwendung von Daten aus Anbieterstatistiken mit Blick auf diese drei Nutzungskontexte. Im Fokus steht der Bereich der öffentlich geförderten Weiterbildung. Nach einer konzeptionellen Verortung im Gesamtsystem der Weiterbildungsstatistik (Abschn. 2) werden die durch das Deutsche Institut für Erwachsenenbildung betreute VHSStatistik und die Weiterbildungsstatistik im Verbund vorgestellt (Abschn. 3). Die Nutzungskontexte Forschung, Politik und Praxis werden erörtert (Abschn. 4) und anhand konkreter Beispiele veranschaulicht (Abschn. 5). Die Revision dieser Statistiken ist ein aktuelles Beispiel für einen koordinierten Abstimmungsprozess zur Modernisierung der Statistik mit Blick auf die verschiedenen Nutzungskontexte. Das erschlossene Nutzungspotenzial wird anhand ausgewählter, modernisierter Merkmale erläutert (Abschn. 6). Im Fazit wird die Revision bewertet und ein Ausblick auf weitere Entwicklungspotenziale gegeben (Abschn. 7). 


\section{Anbieterstatistik im Rahmen der Weiterbildungsstatistik}

Erziehungswissenschaftliche Teilnahmemodelle erklären realisierte Teilnahmen über das Zusammenspiel von Angebot und Nachfrage von und nach Weiterbildung (bspw. Boeren et al. 2010). Die datengestützte Beobachtung und Analyse von Weiterbildung sollte demnach sowohl Merkmale der Nachfrage- als auch der Angebotsseite umfassen. Ausgehend von einer fragmentierten Datenlage können für den Bereich der Weiterbildung vier zentrale Arten von Statistiken differenziert werden: Personenbefragungen, Unternehmensbefragungen, Anbieterstatistiken und Personalstatistiken. Die Anbieterstatistiken bilden demnach eine von vier Säulen einer integrierten Weiterbildungsstatistik (vgl. Kuper et al. 2016, S. 75 ff.). Sie liefern Daten über Struktur und Leistungen von Weiterbildungseinrichtungen. Begrifflich fallen hierunter prinzipiell alle selbstständig und betriebsähnlich organisierten Organisationseinheiten, deren Hauptziel darin besteht, ein Weiterbildungsangebot vorzuhalten (vgl. Pehl 2013, S. 186). Anbieterstatistiken enthalten Daten aus den Meldungen dieser (verbandlich organisierten) Organisationseinheiten und ,haben das Angebotsspektrum und die Belegung der unterbreiteten Angebote zum Thema; zudem lassen sich aus ihnen Angaben zur öffentlichen Finanzierung der Weiterbildung entnehmen" (Kuper et al. 2016, S. 75). Der Bezug auf die öffentliche Finanzierung verweist darauf, dass Statistiken aus Anbieterfeldern, deren Ressourcen und Legitimation vorrangig durch den Kontext Staat oder auch Gemeinschaft geprägt sind (vgl. Schrader 2010a), von besonderem öffentlichen Interesse sind. Sie können Informationsbedarfe im Rahmen gesellschaftlich-politischer Diskussions- und Entscheidungsprozesse über die Erfüllung eines gesellschaftlichen Bildungsauftrages und Ressourcenallokation bedienen und damit zu einer sachlichen bildungspolitischen Diskussion beitragen. Die Weiterbildungsgesetze der Länder sehen vor diesem Hintergrund teilweise die statistische Erfassung von anbieter- und angebotsbezogenen Daten vor (vgl. Pehl 2007, S. 64f.; Ambos et al. 2017, S. 23f.). Im Bereich der öffentlich geförderten Weiterbildung gibt es mit der Volkshochschulstatistik und der Weiterbildungsstatistik im Verbund (siehe Abschn. 4) etablierte Anbieterstatistiken, die mittels systematischer Konzepte Daten zur Struktur, zum realisierten Angebot und zu Teilnahmefällen erheben und eine Relation von Leistungs- und Ressourcendaten (vgl. Schuldt 1998) ermöglichen.

Weitere Datenquellen zu anbieterbezogenen Informationen sind Befragungen von Weiterbildungsanbietern, wobei der wbmonitor hier als einzige kontinuierliche und dem Anspruch nach bundesweit repräsentative Befragung hervorzuheben ist (vgl. Ambos et al. 2018). Die vom Umfang her relativ geringe jährliche Abfrage von Struktur- und Angebotsdaten wird von Schwerpunktbefragungen zu anbieterübergreifend relevanten Themen wie Qualitätsmanagement (2017) oder Personalgewinnung (2014) ergänzt. Die Daten stehen zur wissenschaftlichen Nutzung zur Verfügung. ${ }^{1}$ Die Fernunterrichtsstatistik erhebt auf freiwilliger Basis Daten zu Fernlernangeboten von Bildungsanbietern, die bei der Zentralstelle für Fernunterricht gemeldet sind, sowie von Hochschulen. Auch Anbieter in eher marktförmig geprägten Weiterbildungssegmenten veröffentlichen verbandsintern erstellte Statistiken zu ihren Leistungen (vgl. exemplarisch Wuppertaler Kreis 2017), die jedoch vorran-

\footnotetext{
1 s. https://www.bibb.de/de/1379.php (zugegriffen: 03.12.2018).
} 
gig im kommerziellen Interesse der Unternehmen stehen und in der Regel keinen Anschluss an wissenschaftliche Fragestellungen oder eine Nachnutzung der Daten vorsehen.

\section{Die Anbieterstatistiken am DIE}

Das Deutsche Institut für Erwachsenenbildung - Leibniz-Zentrum für Lebenslanges Lernen (DIE) ist als wissenschaftliches Forschungs- und Serviceinstitut an der Schnittstelle von Weiterbildungspraxis und empirischer Bildungsforschung tätig und wird seit 1990 durch die Leibniz-Gemeinschaft gefördert. Die Durchführung von Anbieterstatistiken und deren Auswertung gehört seit 1962 zum Tätigkeitsbereich des Instituts. Mithilfe zweier Projektförderungen des Bundesministeriums für Bildung und Forschung $(\mathrm{BMBF})^{2}$ wurde der Verbund Weiterbildungsstatistik etabliert, um die Transparenz über Ressourcen und Leistungen der öffentlich geförderten Weiterbildung zu erhöhen (Reichart 2006). Damit wird im Zusammenspiel von freiwilliger Datenlieferung der Verbände und öffentlicher Förderung (Projektförderung und institutionelle Förderung des DIE) ein Beitrag zur Schließung der Informationslücke im Spektrum der Weiterbildungsstatistik (siehe Abschn. 2) geleistet. Zum Verbund Weiterbildungsstatistik gehören aktuell der Bundesarbeitskreis Arbeit und Leben (BAK AL), die Deutsche Evangelische Arbeitsgemeinschaft für Erwachsenenbildung (DEAE) und die Katholische Erwachsenenbildung Deutschland Bundesarbeitsgemeinschaft (KEB). ${ }^{3}$ Der Deutsche Volkshochschul-Verband (DVV) ist assoziiertes Mitglied.

Organisatorisch werden die Statistiken der beteiligten Verbände als je eigene Statistiken geführt, wobei die Liefermodalitäten der Daten, Veröffentlichungsformate, Datennutzungsrechte und Serviceleistungen des DIE jeweils über Kooperationsverträge geregelt sind. Die Daten werden jährlich nach den vereinbarten Merkmalen von den einzelnen datenmeldenden Stellen ${ }^{4}$ über ein Online-Meldeformular an das DIE geliefert, dort in der Abteilung Forschungsinfrastrukturen plausibilisiert, verarbeitet und für die Veröffentlichung und Datenweitergabe aufbereitet. Tab. 1 zeigt die Datenbasis für die teilnehmenden Verbände für das Berichtsjahr 2016.

Die inhaltliche Klammer zwischen den Verbandsstatistiken bildet der vereinbarte sogenannte ,gemeinsame Merkmalskern“ (GMK). Er bezieht sich auf Daten aus den Merkmalsbereichen Institution und Rechtsformen, Personal, Finanzierung und Leistungen. Die Leistungen in Form von Veranstaltungen, Unterrichtstunden und Teilnahmefällen werden nach neun Themenbereichen (bspw. Politik - Gesellschaft, Gesundheit, Sprachen, Arbeit - Beruf und Grundbildung - Schulabschlüsse) diffe-

\footnotetext{
2 „Weiterbildungsstatistik im Verbund“ (2002-2003) und „Transfersicherung Verbund Weiterbildungsstatistik“ (2004-2007).

3 Außer den genannten Verbänden war bis zum Berichtsjahr 2012 auch der Arbeitskreis Deutscher Bildungsstätten (AdB) Mitglied im Verbund.

4 Datenmeldende Stellen sind entweder die einzelnen Einrichtungen selbst oder - weit verbreitet bei den Verbänden BAK AL, DEAE und KEB - übergeordnete Stellen, die die Daten einzelner zugeordneter Einrichtungen zusammenfassen und mittels Sammelbogen weiterleiten.
} 
Tab. 1 Datenbasis im Verbund Weiterbildungsstatistik, Berichtsjahr 2016. (Quelle: Horn et al. 2018, Tab. 1.1)

\begin{tabular}{lcccrc}
\hline & AL & DEAE & DVV & KEB & Insgesamt \\
\hline $\begin{array}{l}\text { Anzahl Mitgliedseinrich- } \\
\text { tungen bundesweit }\end{array}$ & 139 & 456 & 899 & 567 & 2061 \\
Davon ausgewertet & 138 & 355 & 893 & 390 & 1776 \\
Erfassungsquote in \% & 99 & 78 & 99 & 69 & 86 \\
\hline
\end{tabular}

${ }^{a}$ Der Einrichtungsbegriff variiert zwischen den Verbänden; insbesondere im kirchlichen Bereich existieren komplexe institutionelle Strukturen, die die Vergleichbarkeit der Einrichtungszahlen zwischen den Verbänden erschweren (Horn et al. 2018, S. 10).Unter dem Dach der hier aufgeführten VHS-Hauptstellen existieren darüber hinaus sog. Außenstellen, deren Daten über die Hauptstellen gemeldet werden (im Berichtsjahr 2016 bestanden neben 893 ausgewerteten VHS-Hauptstellen 2990 Außenstellen (s. Reichart et al. 2018, Tab. 1)).

renziert und mit Informationen zu Zielgruppen angereichert. Die Erhebungsmerkmale der Verbandsstatistiken gehen in unterschiedlichem Ausmaß über den GMK hinaus, um verbandsspezifische Informationsinteressen $\mathrm{zu}$ bedienen und besondere Struktur- und Leistungsprofile sichtbar zu machen.

Die Ergebnisse zum GMK werden als Open-Access-Publikation veröffentlicht (aktuelle Ausgabe: Horn et al. 2018), ebenso die Volkshochschul-Statistik (Reichart et al. 2018). Des Weiteren werden jährlich verschiedene standardisierte Auswertungen für die innerverbandliche Nutzung zur Verfügung gestellt. Die Daten von BAK AL, der DEAE und der KEB werden ausschließlich als Serviceprodukte für die Verbände (Bundesebene und Landesorganisationen) aufbereitet und präsentiert. Die weitere Veröffentlichung liegt im Ermessen und in der Verantwortung der Verbände. Informationen zu den Volkshochschulen stehen als standardisierte Auswertungen auch für die Fachöffentlichkeit und als Scientific Use File für die Bildungsforschung zur Verfügung (Huntemann 2014).

Unabhängig davon werden vom DIE Daten der Verbandsstatistiken (GMK und Ergebnisse für verbandsspezifische Merkmale) für wissenschaftliche Zwecke der Forschung und Bildungsberichterstattung genutzt und auch Dritten zur Verfügung gestellt (s. Beispiele in Abschn. 5.). Dabei kommen unterschiedliche Bedingungen für die VHS- und die Verbundstatistik zum Tragen. Diese resultieren u.a. aus dem Einsatz von Sammelbögen im Verbund (s.oben), während im VHS-Bereich flächendeckend Daten für die einzelnen Einrichtungen vorliegen. Die Zuordnung von kommunalen VHS zu Gebietskörperschaften lassen für die VHS-Daten auch kleinräumige regionale Analysen im Rahmen von Bildungsberichterstattung, Bildungsmonitoring und empirischer Bildungsforschung zu.

\section{Anforderungen an und Nutzung von Daten aus Anbieterstatistiken}

Die Bereitstellung von aussagekräftigen Anbieterstatistiken für die öffentlich geförderte Weiterbildung ist auf die Zusammenarbeit von Bildungspraxis, Bildungsforschung und Bildungspolitik angewiesen, die jeweils einen spezifischen Beitrag zu diesem Vorhaben beisteuern und ebenso spezifische Interessen an der Datennutzung haben. 
Koordiniert über Verbandsstrukturen sammeln Weiterbildungseinrichtungen in freiwilliger Selbstorganisation Daten, die in der Anbieterstatistik aufbereitet werden. Ihre Bereitschaft zur Mitwirkung und Sorgfalt bei der Datenweitergabe sowie der dafür notwendige Ressourceneinsatz sind essenziell für die Qualität einer Anbieterstatistik. Für die Weiterbildungspraxis gibt es zwei unmittelbare Nutzungskontexte (vgl. Seitter 2013; Pehl 2007, 2013): Nach außen wird die Statistik zum „Sichtbarmachen“, zur Legitimation der eigenen Leistungen und als Nachweis zur wirtschaftlichen Verwendung von (öffentlichen) Mitteln eingesetzt sowie für die Kommunikation in der Öffentlichkeitsarbeit und im Marketing. Nach innen können die aufbereiteten Daten zur selbstreferenziellen Beobachtung und wissensbasierten Steuerung mit dem Ziel der Qualitätssicherung und Organisationsentwicklung genutzt werden, indem bspw. auf Verbandsebene oder auf Einrichtungsebene Entwicklungslinien in den Leistungsdaten nachvollzogen oder Vergleiche anhand bestimmter Referenzpunkte vorgenommen werden (s. unten).

Für die empirische Bildungsforschung ist die Analyse standardisierter Daten eine wichtige Grundlage, um mit interdisziplinären Zugängen Fragen nach den Bedingungen und Möglichkeiten der Bildung Erwachsener im institutionellen und gesellschaftlichen Kontext zu beantworten (vgl. Gräsel 2011). Sie formuliert Standards an die Qualität und Verfügbarkeit von Daten unter den Gesichtspunkten statistischer Gütekriterien, Analyseperspektiven und inhaltlicher Anschlussfähigkeit, die durch wissenschaftliche Begleitung gewährleistet werden. Voraussetzung für die wissenschaftliche Nutzung der Anbieterdaten ist die Anwendbarkeit von deskriptiven und induktiven Methoden - im Rahmen problemorientierter Forschungsvorhaben und auch der Bildungsberichterstattung. Neben dem Anspruch auf Repräsentativität, die durch Vollerhebungen oder Stichprobenverfahren gesichert werden kann, gibt es - abhängig vom Erkenntnisinteresse und den dafür erforderlichen statistischen Modellen - weitere Voraussetzungen. Zum einen ist die Verfügbarkeit der Daten auf Ebene der einzelnen Einrichtung, da bei Aggregatdaten die Gefahr des ökologischen Fehlschlusses besteht (vgl. Gschwend 2006), zu nennen; zum anderen die Verfügbarkeit von kontinuierlichen Zeitreihen bzw. längsschnittlichen Daten, um Veränderungen über die Zeit analysieren zu können.

Während bspw. die schulische und berufliche Bildung durch (halb-)amtliche Statistiken abgedeckt sind, ist dies im Bereich der Weiterbildung nur in wenigen Teilbereichen der Fall (bspw. berufliche Weiterbildung in der Förderstatistik der Bundesagentur für Arbeit). Schon im Strukturplan des Deutschen Bildungsrates wird eine Melde- und Auskunftspflicht von Weiterbildungseinrichtungen und -angeboten diskutiert (Deutscher Bildungsrat 1970, S. 213 f.), die im Bildungsgesamtplan mit dem Ziel des Aufbaus einer bundesweiten Weiterbildungsstatistik konkretisiert wird (BLK 1974, S. 65). Diese und weitere Impulse für eine Weiterentwicklung der Statistik auf gesetzlicher Grundlage (bspw. Expertenkommission Finanzierung Lebenslanges Lernen 2004, S. 286; Bellmann 2003) blieben bis heute ohne Ergebnis. Die Bildungspolitik kann jedoch auch über weniger hierarchische Regelungsstrukturen Entwicklungen der Datengrundlage durch eine strategische Forschungsförderung und die Finanzierung von Infrastrukturprojekten anstoßen. Die DIE-Anbieterstatistiken der öffentlich geförderten Weiterbildung zeigen diese Struktur, indem sie unter Beteiligung von öffentlich finanzierten Forschungsinstituten und mithilfe gezielter 
Projektförderung etabliert und weiterentwickelt werden. Die Nutzenerwartungen einer datengetriebenen und evidenzbasierten Bildungspolitik (vgl. Bellmann 2016) richten sich mit dem Ziel der output-orientierten Steuerung auf Wissen für eine empirisch informierte Bildungsreformpolitik zur Verbesserung der Qualität des Bildungssystems sowie aussagekräftige indikatorenbasierte Monitoringsysteme (Buchhaas-Birkholz 2010), in der Regel in Form der Bildungsberichterstattung, für die zunehmend auch auf regionaler und kommunaler Ebene Bedarf besteht (Schneider et al. 2014). Auf Landesebene gibt es teilweise Statistiken auf Grundlage der Erwachsenen- und Weiterbildungsgesetze (Ambos et al. 2017, S. 23f.). Ein bundesweites Monitoring ist wegen der fehlenden Kompatibilität der verschiedenen Landesstatistiken jedoch nicht möglich. Eine Initiative für eine mögliche Harmonisierung zeichnet sich in den Ländern derzeit nicht $a b$.

Bildungspraxis, -politik und -forschung verbindet das gemeinsame Interesse an einer Datengrundlage, die sich in unterschiedlichen Verwendungskontexten bewähren muss. Ohne an dieser Stelle auf das komplexe Verhältnis und Modi der Kopplung der Funktionssysteme differenziert eingehen zu können (vgl. dazu bspw. Bromme et al. 2016; Bellmann 2006; Terhart 2001; speziell für die Weiterbildung in öffentlicher Verantwortung vgl. Käpplinger 2017), deuten die hier skizzierten spezifischen Nutzenerwartungen bereits an, dass die Anbieterstatistik als gemeinschaftliches Vorhaben das Ergebnis eines Aushandlungsprozesses ist, in dem ein Interessenausgleich zwischen jeweils verschiedenen Handlungslogiken erfolgt. Konkurrierende Interdependenzen bestehen durch spezifische Ressourcenausstattung (Daten, Finanzierung, Expertise) und Nutzenerwartungen. Diese Nutzenerwartungen (Wissen für das Handlungsfeld, Wissen zur Steuerung des Bildungswesens, Analysepotenzial) zeigen mögliche Konfliktlinien auf, weisen jedoch Schnittmengen hinsichtlich der Erwartungen an die Datenqualität und die Transformation von Daten in Steuerungswissen auf. Damit wird die empirische Bildungsforschung zu einem zentralen Bezugspunkt in dieser Dreieckskonstellation.

\section{Beispiele für die Nutzung der DIE-Anbieterstatistiken}

Konkrete Beispiele veranschaulichen im Folgenden die Nutzung der Daten aus den DIE-Anbieterstatistiken in den jeweiligen Kontexten, ohne einen Anspruch auf Vollständigkeit zu erheben.

In bildungswissenschaftlichen Veröffentlichungen gibt es verschiedene Formen der Bezugnahme auf die Anbieterstatistiken. Engels (2018) findet in einer Literaturrecherche 15 Veröffentlichungen, die die VHS-Daten für bildungswissenschaftliche Fragestellungen nutzen, von denen 12 die Daten ausschließlich deskriptiv einsetzen und zwei Publikationen multivariate Verfahren anwenden. Die Analysen können als Teilauswertungen eingebettet in (multimethodische) Forschungsvorhaben (Engels 2018) sein oder sind als Einzelanalyen angelegt (Reichart und Rattinger 2017; Herbrechter und Schemmann 2010; Reichart 2018b). Teilweise werden weitere Daten integriert, bspw. Regionaldaten (Martin und Muders 2017; Martin und Reichart 2019), oder es wird ein Bezug zu qualitativen Daten aus Programmanalysen hergestellt (Fleige und Reichart 2014). Martin und Muders (2017) nutzen die längsschnitt- 
lichen Daten der VHS-Statistik (2000-2013) und beobachten mittels regionalisierter, kausalanalytischer Panelmodellierung, dass in Kooperation durchgeführte Kurse zu zusätzlichen Teilnahmen an Volkshochschulen führen. Allerdings lassen fehlende Informationen zur Qualität der Kooperation und zu soziodemografischen Merkmalen der Teilnehmenden keine differenzierteren Aussagen zu. Engels (2018) ermittelt auf der Grundlage von Differenzen in strukturellen Merkmalen wie Anzahl der Vollzeitäquivalentstellen, Rechtsträger und Finanzierung zwischen zwei Beobachtungszeitpunkten in einer Clusteranalyse typische Strukturprofile von Volkshochschulen, die in einem multimethodischen Forschungsvorhaben für die Fallauswahl im Rahmen einer Interviewstudie genutzt werden. Sie kritisiert undifferenzierte Informationen im Bereich der Finanzierung und des Personals. Martin und Reichart (2019) zeigen mithilfe spatial-autoregressiver Modelle anhand von amtlichen Wahldaten und Umkreisdaten zu Teilnahmefällen an politischer Bildung in Volkshochschulen auf Gemeindeebene, dass das Angebot an politischer Bildung eine positive Wirkung auf die Wahlbeteiligung hat, wobei die Wirkung parteispezifisch variiert. Engels (2018) bewertet die Verwendung der VHS-Daten in der Bildungsforschung zurückhaltend und führt dies unter anderem darauf zurück, dass die VHS-Statistik im Gegensatz zu Primärdaten vorrangig ein Instrument des Bildungsmonitorings ist. Daher sei die Nutzung in bildungswissenschaftlichen Kontexten nur eingeschränkt möglich, da divergierende Interessen zugrunde lägen.

Die Bildungsberichterstattung als Instrument der bildungspolitischen Steuerung stellt über die Daten verschiedene steuerungsrelevante Informationen bereit. Die VHS- und Verbunddaten sind Grundlage für etablierte Berichtssysteme wie den Datenreport des Bundesinstituts für Berufsbildung - BIBB (bspw. Reichart 2018a; Ambos 2018a) und den ,Weiterbildungsatlas“ (Martin und Schrader 2016; Wittenbrink und Frick 2018), in denen die Struktur- und Leistungsdaten bundesweit und regional in Form von Indikatoren und Kennzahlen aufbereitet werden. Gerade in der regionalen Berichterstattung (bspw. Autorengruppe BiLO 2016; Feld et al. 2016; Landesamt für Schulentwicklung/Statistisches Landesamt Baden-Württemberg 2015) sind die Anbieterstatistiken eine wichtige Datenquelle und für das kommunale Monitoring oft die einzige flächendeckend verfügbare Datengrundlage für den Bereich Weiterbildung (Ambos et al. 2017). Sie sind zudem ein wichtiges Element in Überblicksdarstellungen zum gesamten Weiterbildungsbereich (z. B. Deutsches Institut für Erwachsenenbildung 2014) und fließen im Falle der VHSDaten auch in Publikationen des Statistischen Bundesamtes ein (Statistisches Bundesamt 2017). Neben dem Berichtswesen finden sich auch in publizistischen Quellen der politischen Kommunikation Verweise auf die Anbieterdaten, bspw. in Antworten auf parlamentarische Anfragen ${ }^{5}$ oder in Publikationsorganen politischer Akteure, wie Gewerkschaften (Mikschl 2018).

Die Weiterbildungsverbände und -einrichtungen nutzen die Daten vorrangig zur Darstellung der eigenen Leistungen im Rahmen ihres Berichtswesens. Viele Volkshochschulen sowie die Landesverbände und der Bundesverband nutzen die statisti-

\footnotetext{
5 https://kleineanfragen.de/brandenburg/6/9267-die-situation-von-honorarlehrkraeften-in-brandenburgmit-besonderem-fokus-auf-die-dozierenden-in-den; https://kleineanfragen.de/berlin/17/18801-vertragsver haeltnisse-der-vhs-dozentinnen-und-vhs-dozenten.
} 
schen Daten als prominentes Element in ihren Jahresberichten, in Berlin angereichert mit Zusatzdaten (bspw. Thüringer Volkshochschulverband e. V. 2017, S. 26-27; Senatsverwaltung für Bildung, Jugend und Familie 2018; Deutscher VolkshochschulVerband 2018, S. 57-58; Kreisvolkshochschule Groß-Gerau 2017).

Auch der BAK AL und die KEB greifen für ihre regelmäßig erscheinenden Geschäfts- bzw. Jahresberichte auf Daten der Verbandsstatistik zurück (vgl. Bundesarbeitskreis Arbeit und Leben o. J., S. 56f.; KEB 2017, S. 23). Die DEAEVerbandsstatistik und die Verbundstatistik werden außerdem in einer umfassenderen Bildungsberichterstattung verwendet (vgl. Bücker 2018; Bücker und Seiverth 2019), wofür auch Sonderauswertungen der Daten durch das DIE in Anspruch genommen wurden. Weiter finden sich Veröffentlichungen zu spezifischen Themen, bspw. zur Personalsituation und zu Arbeitsbedingungen in der Evangelischen Erwachsenenbildung (Mayert 2017). Neben dem Berichtswesen werden Kennzahlen aus der Statistik auch in der Öffentlichkeitsarbeit genutzt, bspw. in Form von Pressemitteilungen oder in Interviews (VHS Böblingen-Sindelfingen 2018; ZDF 2017) sowie in der Kommunikation mit politischen Entscheidungsträgern (Lindemann 2017). Weber (2017) beschreibt mit Bezug auf die neue Kategorie berufsbezogener Veranstaltungen (s. unten), dass statistische Kategorien in verschiedenen Verwendungskontexten der VHS (z. B. Steuerberechnung für einzelne VHS durch das Finanzamt, Darstellung der Rolle der VHS in der Gesellschaft) unterschiedliche Bedeutung haben bzw. dieselben Ergebnisse in diesen Kontexten unterschiedlich bewertet werden können.

Für die interne Steuerung werden in der Verbandsarbeit Kennzahlen definiert, die die Volkshochschulen in Benchmarking- und Strategieprozessen nutzen können (z.B. Huba und Zierer 2007; Reichart 2012; Fiebig 2016; Pehl 2007).

\section{Revision der Anbieterstatistik}

Etwa seit der Jahrtausendwende haben sich die Anforderungen an und das Leistungsspektrum von Weiterbildungseinrichtungen gewandelt: Seit den Lernenden mit Blick auf das Paradigma des Lebenslangen Lernens verstärkt die Verantwortung für ihre individuellen Bildungsprozesse zugeschrieben wird, übernehmen Weiterbildungseinrichtungen zunehmend auch beratende Funktionen und stellen Infrastrukturen für das Selbstlernen bereit. Bildungspolitisch wichtige Themen, wie Digitalisierung, Grundbildung und Integration von Flüchtlingen, wurden von den Einrichtungen in ihrem Angebotsspektrum aufgegriffen, konnten aber mit der bisherigen Statistik nicht adäquat dargestellt werden. Insofern waren viele - auch politisch erwünschte - Arbeitsbereiche der Einrichtungen untererfasst bzw. gar nicht statistisch repräsentiert. Aus wissenschaftlicher Perspektive des DIE kam der Bedarf hinzu, offenkundig gewordene Inkonsistenzen der Statistik zu beseitigen (s. unten GMK). Dazu kam das Interesse der teilnehmenden Weiterbildungsorganisationen, ihre verbandsspezifischen Angebotsprofile besser als bisher sichtbar zu machen. Dies deckt sich mit dem wissenschaftlichen und bildungspolitischen Bedarf, die Pluralität der Weiterbildungslandschaft (Schrader 2010b) statistisch adäquat abzubilden (s. Abschn. 2).

Im Projekt „Große Revision der DIE-Anbieter-/Angebotsstatistiken - StaRe“ (Laufzeit: 2014-2018; gefördert durch das BMBF) wurden die statistische Erhe- 
bung und standardisierten Ergebnisdarstellungen der beteiligten Verbände (siehe Abschn. 4) mit folgenden Zielen revidiert:

- Abbildung neuer Lerndienstleistungen

- Modernisierung und Ergänzung der bisherigen Systematik der Erfassung von Einrichtungs-, Personal-, Finanz- und Veranstaltungsdaten

- Bessere Sichtbarkeit verbandsspezifischer Angebotsprofile

- Anschlussfähigkeit an aktuelle und zukünftige bildungspolitische Anforderungen

- Erhalt eines gemeinsamen Merkmalskerns der vier Verbände

Angestrebt war, die adäquate und umfassende Darstellung der Leistungen der Weiterbildungsanbieter mit größtmöglicher Kontinuität der Berichterstattung zu verbinden. Durch die umfassende Aktualisierung sind die Daten vor und nach der Revision jedoch nur noch eingeschränkt konsistent, sodass Zeitreihen aus surveymethodischen Gründen durch das DIE vorerst nicht veröffentlicht werden. Im Rahmen eines dreijährigen Moratoriums wird geprüft werden, für welche Merkmale bzw. Merkmalsaggregate Zeitreihen über den Bruch der Revision hinweg geführt werden können (AK BBE 2018).

Da es sich bei den Anbieterstatistiken des DIE um freiwillige Statistiken handelt (siehe oben), war es zielführend, die Revision in einem dialogischen Verfahren zwischen dem DIE als wissenschaftlicher Serviceeinrichtung und den Vertreterinnen und Vertretern der Verbände und verschiedenen Verbandsgremien zu gestalten (s. oben Abschn. 3 und zur Projektstruktur Schön 2014). Damit wurde sichergestellt, dass die zu erhebenden Merkmale praxisrelevant, verständlich und gegenstandsangemessen sind. Zugleich konnte so die Akzeptanz der Änderungen bei den datenmeldenden Stellen gefördert werden.

Den GMK betreffende Modifizierungen wurden gemeinsam diskutiert und nach dem Konsensprinzip entschieden. Prüfkriterien und Abwägungsprozesse orientierten sich jeweils an dem Informationsbedarf der Verbände, dem potenziellen Nutzen der erhobenen Informationen für die Bildungsberichterstattung und dem Aufwand, den die Erfassung des jeweiligen Merkmals für die datenmeldenden Stellen voraussichtlich mit sich bringen würde.

Eingesetzt werden die revidierten Merkmale im Verbund erstmals für das Berichtsjahr 2017, in der VHS-Statistik für 2018. Im Folgenden werden ausgewählte Beispiele zunächst aus dem revidierten GMK und anschließend aus dem Spektrum der neuen oder veränderten verbandsspezifischen Merkmale vorgestellt. Dabei wird auch erläutert, in welcher Hinsicht ein Mehrwert an Informationen zu erwarten ist. Dies betrifft verbandsinterne Zwecke der internen Steuerung, die Legitimation oder die Außendarstellung von Leistungen und - v. a. im Bereich des GMK - die Bildungsberichterstattung. Auf Potenziale für den Nutzungskontext Bildungsforschung wird abschließend gebündelt eingegangen.

\subsection{Gemeinsamer Merkmalskern (GMK)}

Tab. 2 gibt einen Überblick über die zukünftigen zum GMK gehörenden Erhebungsmerkmale und Hinweise auf Art und Umfang der im Rahmen der Revision vorgenommenen Änderungen. 
Tab. 2 Gemeinsamer Merkmalskern der Weiterbildungsstatistik im Verbund nach der Revision

\begin{tabular}{|c|c|}
\hline Merkmale & Art und Umfang der Änderungen \\
\hline \multicolumn{2}{|l|}{ Institutionelle Daten } \\
\hline Rechtsform (Träger bzw. Einrichtung) & Zusätzliche Ausprägung \\
\hline Qualitätsmanagementsystem(e) & Neues Merkmal \\
\hline Anerkennung nach Erwachsenen-/Weiterbildungsgesetz & Neues Merkmal \\
\hline $\begin{array}{l}\text { Durchführung von Veranstaltungen mit Anerkennung nach } \\
\text { einem Bildungsfreistellungsgesetz }\end{array}$ & Neues Merkmal \\
\hline \multicolumn{2}{|l|}{ Personal } \\
\hline $\begin{array}{l}\text { Stellen (Vollzeitäquivalente; differenziert nach Frauen und } \\
\text { Männern) }\end{array}$ & Zusätzliche Ausprägungen \\
\hline Personen (Anzahl, differenziert nach Frauen und Männern) & Differenzierung der Ausprägungen \\
\hline \multicolumn{2}{|l|}{ Finanzierung } \\
\hline Einnahmen (differenziert nach Einnahmenquellen) & Zusätzliche Ausprägung \\
\hline Ausgaben (differenziert nach Ausgabearten) & Keine Änderungen \\
\hline \multicolumn{2}{|l|}{ Veranstaltungen } \\
\hline $\begin{array}{l}\text { Veranstaltungen (Kategorien: i.d.R. mit mehr als bzw. mit } \\
\text { bis zu drei Veranstaltungsstunden; differenziert nach Anzahl } \\
\text { der Veranstaltungen, Veranstaltungsstunden, Teilnahmen) }\end{array}$ & $\begin{array}{l}\text { Differenzierung in berufsbezogene } \\
\text { und (weitere) nicht berufsbezogene } \\
\text { Veranstaltungen } \\
\text { Modifizierung der Themenbereiche } \\
\text { (nicht berufsbezogene Veranstaltungen) }\end{array}$ \\
\hline $\begin{array}{l}\text { Veranstaltungen mit digital vermittelten Lerninhalten (An- } \\
\text { zahl) }\end{array}$ & Neues Merkmal \\
\hline $\begin{array}{l}\text { Veranstaltungen für spezielle Adressatengruppen (Anzahl, } \\
\text { differenziert nach berufsbezogenen und nicht berufsbezoge- } \\
\text { nen Veranstaltungen nach Themenbereichen) }\end{array}$ & Zusätzliche Ausprägungen \\
\hline \multicolumn{2}{|l|}{ Weitere Leistungen } \\
\hline $\begin{array}{l}\text { Weiterbildungsberatung (Anzahl Beratene und Beratungs- } \\
\text { stunden) }\end{array}$ & Neues Merkmal \\
\hline $\begin{array}{l}\text { Kompetenzfeststellung } \\
\text { Prüfungen und Tests } \\
\text { Kompetenzerfassungen/-bilanzierungen } \\
\text { (Teilnahmen) }\end{array}$ & Neues Merkmal \\
\hline
\end{tabular}

\subsubsection{Merkmalsbereich Finanzierung/Einnahmen: Öffentliche Mittel - eingeworbene Mittel}

Für die Abbildung der Einnahmen der Einrichtungen aus öffentlichen Mitteln wurde im GMK die Differenzierung nach verschiedenen Geldgebern beibehalten (EU, Bund, SGB, Land, Kommune), aber ergänzt um den Anteil eingeworbener Mittel an allen öffentlichen Mitteln (als Davon-Kategorie). Die Aufnahme dieser v. a. für bildungspolitische Zwecke auf Verbandsebene wichtigen Information trägt den Erfahrungen der Praxis Rechnung, wonach die öffentliche Förderung in der jüngeren Vergangenheit in zunehmendem Maße durch die Beteiligung an Ausschreibungen und zeitlich befristeten Förderprogrammen erfolgt und nicht unerhebliche Personalressourcen in den Einrichtungen bindet. 


\subsubsection{Merkmalsbereich Veranstaltungen: Berufsbezogene Veranstaltungen}

Im revidierten GMK werden berufsbezogene Veranstaltungen separat erfasst. Damit werden zum einen Inkonsistenzen in Bezug auf die Zuordnung von Veranstaltungen zum bisherigen Themenbereich Arbeit - Beruf beseitigt. ${ }^{6}$ Zum anderen werden vereinzelt bei den Verbänden bestehende, v. a. bildungspolitisch motivierte Interessen an der expliziten Erfassung berufsbezogener Angebote aufgegriffen.

Hierdurch eröffnet sich für die beteiligten Verbände die Möglichkeit, den Stellenwert beruflicher Weiterbildung im Angebotsprofil ihrer Einrichtungen zukünftig besser datengestützt sichtbar machen zu können und für Steuerungszwecke zu nutzen (vgl. ausführlich Ambos 2017).

Auch in der Bildungsberichterstattung hat die Abgrenzung von beruflicher und nicht-beruflicher Weiterbildung einen hohen Stellenwert, bspw. beim Adult Education Survey, der umfangreichsten Personenbefragung zur Weiterbildungsbeteiligung (vgl. Bilger et al. 2017). Insofern ist auch für dieses Feld ein Mehrwert durch die explizite Ausweisung berufsbezogener Weiterbildung zu erwarten. Zum Beispiel werden für den Datenreport zum Berufsbildungsbericht des BIBB, für den das DIE regelmäßig Beiträge zur beruflichen Weiterbildung an VHS und in Einrichtungen der am Verbund beteiligten Verbände liefert, zukünftig validere Daten berichtet werden können. Bisher wurden Näherungswerte dargestellt, die auf Auswertungen zum Themenbereich „Arbeit - Beruf“ basierten (Ambos 2018a; Reichart 2018a).

\subsubsection{Merkmalsbereich Veranstaltungen: Themenbereich Alphabetisierung - Grundbildung}

Ein Zugewinn an Informationen zu den thematischen Angebotsprofilen der durch die VHS- und Verbundstatistik erfassten Bereiche der öffentlich verantworteten Weiterbildung ist auch von der separaten Ausweisung von Veranstaltungen im Themenbereich Alphabetisierung - Grundbildung zu erwarten. Die bildungspolitische Bedeutung entsprechender Angebote für Erwachsene belegt aktuell u. a. die „Nationale Dekade für Alphabetisierung und Grundbildung“, in deren Rahmen Bund, Länder und weitere Partner eine Verbesserung der Grundbildung in Deutschland anstreben (Näheres s. unter https://www.alphadekade.de/index.html). Zugleich ist die Datenlage in diesem Feld als defizitär zu betrachten, auch weil die Gewinnung bundesweiter Daten mit besonderen Herausforderungen einhergeht (vgl. Ambos 2018b). Insofern stellt die Trennung vom Themenbereich „Schulabschlüsse“ ein wichtiges Ergebnis der Revision dar. Dies gilt mit Blick auf den dadurch besseren Nachweis

\footnotetext{
${ }^{6}$ Der Themenbereich (in der VHS-Statistik: Programmbereich) Arbeit - Beruf umfasste in der Verbundstatistik Veranstaltungen, die von ihrem Inhalt oder ihrer didaktischen Ausrichtung her einen Berufsbezug aufwiesen. Inhaltlich wurden hier einerseits PC-Kurse und technische bzw. kaufmännische Veranstaltungen einsortiert (v. a. in der VHS-Statistik), aber auch Veranstaltungen zum Arbeitsleben, z. B. Interessenvertretung oder zu Soft Skills. In der ansonsten nach Inhalten aufgebauten Statistik ergab sich dadurch eine Inkonsistenz, die in der revidierten Statistik dadurch behoben wird, dass die Themenbereiche inhaltlich gefüllt werden und eine zusätzliche Differenzierung nach dem Berufsbezug aufgenommen wird (s. ausführlich Ambos 2017).
} 
von Leistungen in diesem Feld auf Seiten der Weiterbildungspraxis wie auch in der Bildungsberichterstattung.

\subsubsection{Merkmalsbereich Weitere Leistungen: Weiterbildungsberatung}

Ein zentrales Motiv der beteiligten Verbände für die Revision war die Abbildung bisher nicht erfasster lernbezogener Dienstleistungen, durch die die Einrichtungen in den letzten Jahren ihr Angebotsportfolio vielfach erweitert haben. Dazu zählen verbandsübergreifend u.a. Beratungsleistungen für an Weiterbildung interessierte Personen. Mit der Ausweisung von Quantitäten zu Beratenen und Beratungsstunden im Feld der Weiterbildungsberatung im GMK werden erstmals datengestützte Informationen dazu vorliegen. Eine besondere Bedeutung haben derartige Informationen nicht zuletzt auch vor dem Hintergrund, dass derartige Beratungsleistungen zwar politisch gefordert werden, auf Basis der geltenden Erwachsenen- und Weiterbildungsgesetze der Länder bisher aber nicht allgemein förderfähig sind. Durch eine zusätzliche Abfrage ist es möglich, auf Basis von gesetzlichen Regelungen und/ oder Programmen von Bund und/oder Ländern erbrachte Beratungsleistungen zur Förderung/Finanzierung von Weiterbildung gesondert sichtbar zu machen.

\subsection{Verbandsspezifische Merkmale (exemplarisch)}

Auch nach der Statistikrevision gilt, dass die spezifischen Angebotsprofile und Strukturen der beteiligten Verbände über verbandsspezifische Erhebungsmerkmale in besonderer Weise statistisch abbildbar sein sollen. Entsprechende Verbesserungen zu erreichen war daher ebenfalls ein wichtiges Ziel der Revision.

\subsubsection{BAK AL: Merkmalsbereich Weitere Leistungen, Beratungen von verschiedenen Adressaten und Organisationen zu diversen Themen}

Neben der Beratung von an Weiterbildung interessierten Personen (s.oben) haben im Leistungsprofil der Einrichtungen von Arbeit und Leben in den letzten Jahren auch Beratungen von anderen Adressatinnen und Adressaten sowie Organisationen an Bedeutung gewonnen, die in der bisherigen Verbandsstatistik jedoch nicht abgebildet wurden. Um hier datengestützt Transparenz herzustellen, werden nach der Revision Beratungsleistungen für individuell Ratsuchende im Bereich Arbeitsrecht, Antidiskriminierung u. ä. sowie Beratungsleistungen für betriebliche Interessenvertretungen, Betriebe und andere Institutionen/Multiplikatoren $\mathrm{zu}$ jeweils einschlägigen Themenbereichen erhoben.

\subsubsection{DEAE: Merkmalsbereich Veranstaltungen, Qualifizierungsangebote zum ehrenamtlichen, kirchlichen und zivilgesellschaftichen Engagement}

Profilbildend für die DEAE-Einrichtungen ist u.a. das Segment der Qualifizierung von Personen, die sich ehrenamtlich, zivilgesellschaftlich oder kirchlich engagieren. Die Zuordnung derartiger Veranstaltungen zu bestehenden Themenbereichen hatte sich für den Verband als unzureichend und reformbedürftig erwiesen. Zukünftig 
werden solche Qualifizierungen in einer eigenen Kategorie erhoben - analog zur Erfassung von berufsbezogenen Veranstaltungen (s. oben GMK).

\subsubsection{DVV: Merkmalsbereich Veranstaltungen, Integrationskurse}

Der DVV ist der mit Abstand größte Anbieter von Integrationskursen (Bundesamt für Migration und Flüchtlinge 2017, S. 17); mehr als die Hälfte der Volkshochschulen waren 2016 als Träger zugelassen. Vor der Revision wurden die Integrationskurse zusammen mit anderen Deutschkursen im Fachgebiet Deutsch als Fremdsprache gezählt, waren dort aber nicht einzeln identifizierbar. In der revidierten VHS-Statistik werden Integrationskurse separat erfasst, wobei Integrationskurse mit dem Schwerpunkt Alphabetisierung zusätzlich ausgewiesen werden. Zusammen mit der ebenfalls neu eingeführten differenzierten Erfassung der Finanzierungsquellen, die eine separate Ausweisung von Mitteln des Bundesamts für Migration und Flüchtlinge sowie von sonstigen Mitteln zur Sprachförderung vorsieht, wird die Statistik künftig Informationen zu Umfang und Ressourcen in diesem bildungspolitisch bedeutsamen und aktuellen Arbeitsfeld der Volkshochschulen bereitstellen. ${ }^{7}$

\subsubsection{DVV: Merkmalsbereich Weitere Leistungen, Betreuungs- und Unterstützungsleistungen}

Neben den klassischen Weiterbildungsveranstaltungen und der Weiterbildungsberatung sind die Volkshochschulen seit einigen Jahren auch in weiteren Arbeitsfeldern tätig, die bisher über die Statistik nicht erfasst wurden: Sie sind in einigen Bundesländern als Anbieter offener Ganztagsangebote für Schulkinder aktiv und bieten darüber hinaus Betreuungsangebote für Kinder von Weiterbildungsteilnehmenden oder auch sozialpädagogische Betreuung von Weiterbildungsteilnehmenden an. Diese Angebote sowie Leistungen zur Unterstützung bei der Vermittlung in Arbeit und Maßnahmen der Lernförderung (z. B. über das sog. Bildungs- und Teilhabepaket) werden künftig nach ihren Betreuungs-/Beratungs- bzw. Unterrichtsstunden und nach Teilnahmefällen erfasst. Damit werden die Leistungen der Volkshochschulen über ein klassisches Kursangebot hinaus verdeutlicht.

\subsubsection{KEB: Merkmalsbereich Veranstaltungen, Veranstaltungen für besondere Adressatengruppen}

Veranstaltungen für besondere Adressatengruppen werden verbandsübergreifend erhoben und im GMK ausgewiesen. Darüber hinaus haben die Verbände die Möglichkeit, für die Herausstellung ihrer spezifischen Leistungen zusätzlich Daten zu für sie wichtigen ,eigenen“ Kategorien zu erheben. Davon hat u.a. die KEB Gebrauch gemacht: Nach der Revision wird bei der Abfrage die Ausprägung „Ehrenamtli-

\footnotetext{
7 Aufgrund der hohen bildungspolitischen Bedeutung dieses Themas wurde dieses Erhebungsmerkmal bei einem Großteil der VHS bereits ab dem Berichtsjahr 2016 im Rahmen einer Zusatzerhebung abgefragt (s. für die Ergebnisse Reichart et al. 2018, S. 93-103).
} 
che“ neu aufgenommen; dieser Adressatengruppe wird neben „Familien“ besondere Relevanz für das KEB-Angebotsprofil zugewiesen.

\subsection{Analysepotenzial für die empirische Bildungsforschung}

Ein erweitertes Analysepotenzial durch die Statistikrevision besteht insbesondere auf der Ebene von Einzeleinrichtungen. Aufgrund der weiterhin geltenden strukturellen Unterschiede zwischen VHS- und Verbundstatistik (s.oben Abschn. 3) werden die Möglichkeiten im Bereich des Verbundes begrenzt bleiben. Zusätzliche Perspektiven eröffnen sich vor allem für die VHS-Daten, bspw. mit Blick auf Fragestellungen zum Angebotsprofil und zum Leistungsspektrum der Einrichtungen in Abhängigkeit von regionalen oder bildungspolitischen Rahmenbedingungen. Dies könnte z. B. mit Blick auf Veranstaltungen in den Bereichen der berufsbezogenen Weiterbildung oder Alphabetisierung-Grundbildung von Interesse sein. Zu beachten ist hierbei, dass die Zuordnung zu den statistischen Kategorien in der einzelnen VHS erfolgt und i.d.R. durch die Programmplanenden vorgenommen wird (vgl. mit Blick auf berufsbezogene Veranstaltungen Weber 2017, S. 284). Durch klare definitorische Vorgaben, die in Zusammenarbeit zwischen DIE und DVV erarbeitet werden, wird dabei eine möglichst einheitliche Handhabung der Kategorien sichergestellt. Letztendlich wird die Datenqualität durch eine umfangreiche und umfassende Plausibilisierung der gemeldeten Daten im DIE sichergestellt. Im Berichtsjahr 2017 haben 882 Volkshochschulen Daten gemeldet. Aufgrund von aufgefallenen Diskrepanzen zu den Vorjahresdaten wurden im Rahmen der Plausibilisierung 308 Volkshochschulen angerufen (ca. $35 \%$ ). Bei 225 Volkshochschulen wurde im Zuge der Plausibilisierung mindestens ein Datenfeld korrigiert.

Erweiterungen oder Modifikationen einzelner Merkmale, etwa zu Kooperationspartnern bei der Durchführung von Kursen oder Altersgruppen der Teilnehmenden, gehen direkt auf bereits geäußerte Bedarfe an die Statistik (Abschn. 5) ein. Das Paneldesign der VHS-Statistik ist durch die wiederholte Messung der Merkmale nicht nur für die Beobachtung von Trends geeignet (bspw.: Wie entwickelt sich die Zahl der Integrationskurse?), sondern prinzipiell auch für Fragestellungen, die sich auf kausale Zusammenhänge beziehen (bspw.: Welche Auswirkung hat die Entwicklung im Bereich der Integrationskurse auf die Entwicklung in anderen Programmbereichen?). Allerdings sind das längsschnittliche Analysepotenzial sowie die Beschreibung von Trends in der Berichterstattung durch das umfassend revidierte Erhebungskonzept zunächst zurückgeworfen. Inwiefern die Statistik vor und nach der Revision eine Fortschreibung von Merkmalen ermöglicht, muss in den kommenden Jahren auf Merkmalsebene empirisch geprüft werden (AK BBE 2018).

\section{Fazit und Perspektiven}

Eine träger- und bundesländerübergreifende Statistik zu Struktur-, Leistungs- und Teilnahmedaten von Weiterbildungsanbietern in freiwilliger Selbstverantwortung ist eine beachtliche Leistung der Weiterbildungsverbände in öffentlicher Trägerschaft, zumal sie sich in weiten Teilen an den Qualitätsstandards amtlicher Statistiken (vgl. 
Eurostat und Europäisches statistisches System 2018) orientiert, ohne über deren verpflichtende juristische bzw. amtliche Rahmung sowie Ressourcen zu verfügen. Die Wissenschaft, hier in Vertretung des DIE, betreut den Data Life Cycle von der Datenerhebung über die Qualitätssicherung bis zur Bereitstellung, finanziert durch die institutionelle Förderung des DIE sowie über projektbezogene Mittel des BMBF für spezifische Entwicklungsprojekte. Diese Konstellation führt zu einer hohen Transparenz über Leistungen der Weiterbildung in öffentlicher Trägerschaft. Die wissenschaftliche Begleitung gewährleistet einerseits die Qualität der Erhebung und stellt andererseits die Expertise für Auswertungen und Interpretationen der Daten bereit, mit denen anwendungsbezogen verschiedene Bedarfe in Praxis und Politik aufgegriffen werden können.

Für die Bildungsforschung sind die Anbieterstatistiken am DIE eine zentrale und in dieser Qualität einzigartige Datengrundlage für Fragen, die sich auf die institutionellen Strukturen des Lernens Erwachsener beziehen. Mit der Revision ist es in großen Teilen gelungen, die Entwicklungen und Anforderungen in diesem Bereich durch die Weiterentwicklung des statistischen Beobachtungsrasters adäquat abzubilden, was notwendigerweise mit Brüchen in der Kontinuität von Zeitreihen einhergeht. Bemerkenswert ist, dass sich dieses Update neben Neuordnungen und der weiteren Differenzierung im Rahmen des traditionellen veranstaltungsbezogenen Angebots zu einem großen Teil auch durch die Neuaufnahme von Beratungs-/ Betreuungsleistungen auszeichnet. Für die erfolgreiche Fortschreibung und Weiterentwicklung ist die Berücksichtigung von Schnittmengen der drei Nutzungskontexte Forschung, Politik und Praxis weiterhin zentral.

Zur Bewertung des Nutzens müssen auch die aufgebrachten Ressourcen berücksichtigt werden. Während der Nutzen für die Praxis aus Sicht der Verbände unstrittig ist, stellt sich das Verhältnis von Aufwand und Nutzen für die Einzeleinrichtungen teilweise unausgewogen dar. Um Potenziale auf Ebene der Einzeleinrichtungen sichtbar zu machen, könnten Möglichkeiten der Nutzung der Daten auf Einrichtungsebene im Rahmen der strategischen Planung und Außenkommunikation verdeutlicht und in kooperativen Projekten Bedarfe der Praxis identifiziert und Lösungen entwickelt werden, die durch moderne digitale Werkzeuge der Datenaufbereitung und -visualisierung die Datennutzung fördern. Hilfreich für die Gewährleistung eines direkten Nutzens auf Einrichtungs- und Verbandsebene wäre ebenso eine höhere Kompatibilität zu den verpflichtenden Leistungsstatistiken auf Landesebene. Hierfür bräuchte es aufgrund der föderalen Strukturen eine entsprechende Willensbekundung und Koordination auf Ebene der KMK, die sich allerdings bisher nicht abzeichnet (vgl. Ambos 2008).

Das Analysepotenzial der Daten wächst mit Blick auf die Anbieter bzw. Weiterbildungssegmente, zu denen Daten vorliegen. Eine Expansion der Anbieterstatistik, bspw. durch das Vorhaben einer Statistik zur wissenschaftlichen Weiterbildung an Hochschulen, wie sie aktuell in Kooperation mit der Deutschen Gesellschaft für wissenschaftliche Weiterbildung und Fernstudium (DGWF) geplant ist (Wolter et al. 2019), erhöht allgemein die Sichtbarkeit und ermöglicht empirisches Wissen zum pluralen Weiterbildungsbereich. Darüber hinaus ist das Analysepotenzial in hohem Maße von der Qualität und auch des Aggregationsgrads der Daten abhängig. Deutlich wird dies daran, dass sich die wissenschaftlichen Analysen fast 
ausschließlich auf die VHS-Statistik beziehen, die als Einzige Daten durchgehend auf Einrichtungsebene zur Verfügung stellt. Eine vergleichbare Auflösung bei den weiteren beteiligten Verbänden wäre ein erstrebenswertes langfristiges Ziel für die Weiterentwicklung der Anbieterstatistiken.

Des Weiteren könnte geprüft werden, unter welchen Voraussetzungen eine Erhebung auf Veranstaltungsebene nutzbringend und für die Einrichtungen realisierbar ist, da auf dieser Analyseebene differenziertere Fragestellungen und Beobachtungen möglich sind, bspw. zu Fragen der mesodidaktischen Planung oder Nutzung spezifischer Veranstaltungsformate in Abhängigkeit soziodemografischer Merkmale der Teilnehmenden. Unmittelbar bevor steht jedoch die Prüfung der revidierten Daten hinsichtlich ihrer Qualität und Anschlussmöglichkeiten an das vor der Revision bestehende Erhebungskonzept. Die erstmalige Veröffentlichung der revidierten Daten ist für den Verbund Weiterbildungsstatistik ohne DVV (zum Berichtsjahr 2017) sowie die Volkshochschul-Statistik (zum Berichtsjahr 2018) im Jahr 2019 geplant.

Im Zuge des Revisionsprojekts kommt es außerdem zu einer zielgruppenbezogenen Weiterentwicklung der begleitenden Produkte. Die Berichte (Verband und VHS) werden mit Blick auf Struktur und Aufbereitung der Ergebnisse grundlegend überarbeitet; zusätzliche Elemente, bspw. in Form eines Glossars oder Informationen zur Vergleichbarkeit der institutionellen Rahmenbedingungen auf Ebene der Bundesländer, werden die Transparenz erhöhen und es den Nutzenden ermöglichen, die Qualität der Ergebnisse besser einzuschätzen. Der Scientific Use File der VHS-Statistik wird mit Blick auf Format und Dokumentation entsprechend den Standards sozialwissenschaftlicher Forschungsdaten überarbeitet. Die Anreicherung des SUF mit beschreibenden Metadaten, die Aufbereitung des Datenhandbuchs für Forschende sowie die Listung des SUF beim Verbund Forschungsdaten Bildung (https://www. forschungsdaten-bildung.de) sind die ersten wichtigen Schritte, die Sichtbarkeit der Daten zu erhöhen und die wissenschaftliche Nutzung zu fördern.

\section{Quellen im Internet}

- AlphaDekade 2016-2026: https://www.alphadekade.de/index.html.

- Revidierter VHS-Berichtsbogen: https://www.die-bonn.de/weiterbildung/statistik/ vhs-statistik/VHS_Berichtsbogen2.pdf.

- Verbund Forschungsdaten Bildung: https://www.forschungsdaten-bildung.de

Open Access Dieser Artikel wird unter der Creative Commons Namensnennung 4.0 International Lizenz (http://creativecommons.org/licenses/by/4.0/deed.de) veröffentlicht, welche die Nutzung, Vervielfältigung, Bearbeitung, Verbreitung und Wiedergabe in jeglichem Medium und Format erlaubt, sofern Sie den/die ursprünglichen Autor(en) und die Quelle ordnungsgemäß nennen, einen Link zur Creative Commons Lizenz beifügen und angeben, ob Änderungen vorgenommen wurden.

\section{Literatur}

AK BBE, Arbeitskreis Bildungsberichterstattung am DIE (2018). Moratorium für Zeitreihenanalysen der VHS-Statistik. Bonn: Deutsches Institut für Erwachsenenbildung. Online-Erstveröffentlichung, Stand Informationen: Mai 2018; https://www.die-bonn.de/id/36848. Zugegriffen: 11.04.2019 
Ambos, I. (2008). Mehr Vergleichbarkeit in der institutionellen Weiterbildungsstatistik. Dokumentation der Veranstaltung am 8. und 9. April 2008 in Köln, Bonn. https://www.die-bonn.de/doks/ambos0801.pdf. Zugegriffen: 3. Dez. 2018.

Ambos, I. (2017). Die Abbildung beruflicher Weiterbildung in der Volkshochschul- und der Verbundstatistik - Aktueller Stand und Perspektiven nach der Revision der DIE-Anbieter-/Angebotsstatistiken. Hessische Blätter für Volksbildung, 3, 257-265.

Ambos, I. (2018a). Weiterbildungsstatistik im Verbund. In Bundesinstitut für Berufsbildung (Hrsg.), Datenreport zum Berufsbildungsbericht 2018: Informationen und Analysen zur Entwicklung der beruflichen Bildung (1. Aufl. S. 382-385). Bonn: Barbara Budrich. Kap. B 2.2.3; https://nbn-resolving.de/ urn:nbn:de:0035-0729-4. Zugegriffen: 10.12.2018.

Ambos, I. (2018b). Herausforderungen der Berichterstattung zur Alphabetisierung/Grundbildung Erwachsener am Beispiel alphamonitor. In M. Lüdtke \& B. Schulz (Hrsg.), Wie wirkt Grundbildung? Eine Zwischenbilanz des Projekts eVideoTansfer (1. Aufl. S. 40-46). Berlin: ARBEIT UND LEBEN DGB/VHS. https://www.lernen-mit-evideo.de/wp-content/uploads/2019/02/eVideo_240119_WEB. pdf\#page=40. Zugegriffen: 11.04.2019.

Ambos, I., Koscheck, S., Martin, A., \& Reuter, M. (2018). Qualitätsmanagementsysteme in der Weiterbildung. Ergebnisse der wbmonitor Umfrage 2017, Bonn. https://wbmonitor.bibb.de/downloads/ Ergebnisse_20180507.pdf. Zugegriffen: 30. Aug. 2018.

Ambos, I., Middendorf, L., \& Weiß, C. (2017). Handreichung. Weiterbildung als Gegenstand des kommunalen Bildungsmonitorings, Bonn. https:/www.transferinitiative.de/media/content/DLR_ Weiterbildung.pdf. Zugegriffen: 14. Dez. 2018.

Autorengruppe Bi, L. (2016). Atlas Bildungslandschaft Oberfranken. Herausgeber: Leibniz Institut für Bildungsverläufe e. V. Bamberg. Www.bilo-Atlas.de. https://doi.org/10.5157/BiLO:Atlas.

Bellmann, J. (2006). Bildungsforschung und Bildungspolitik im Zeitalter ,Neuer Steuerung‘. Zeitschrift für Pädagogik, 52(4), 487-504.

Bellmann, J. (2016). Datengetrieben und/oder evidenzbasiert? Wirkungsmechanismen bildungspolitischer Steuerungsansätze. Zeitschrift für Erziehungswissenschaft, 19(Suppl 1), 147-161.

Bellmann, L. (2003). Datenlage und Interpretation der Weiterbildung in Deutschland. Bielefeld: W. Bertelsmann.

Bilger, F., Behringer, F., Kuper, H., \& Schrader, J. (Hrsg.). (2017). Weiterbildungsverhalten in Deutschland 2016. Ergebnisse des Adult Education Survey (AES). DIE Survey. Bielefeld: W. Bertelsmann.

BLK (Bund-Länder-Kommission für Bildungsplanung) (1974). Bildungsgesamtplan. Bd. I. Stuttgart: Klett.

Böblingen-Sindelfingen, V.H.S. (2018). Volkshochschule Böblingen-Sindelfingen. VHS gefragt wie noch nie - neues vhs-Programm liegt aus. FOCUS Online vom 11.01.2018. https://www.focus.de/ regional/baden-wuerttemberg/volkshochschule-boeblingen-sindelfingen-vhs-gefragt-wie-noch-nieneues-vhs-programm-liegt-aus_id_8286316.html. Zugegriffen: 24. Okt. 2018.

Boeren, E., Nicaise, I., \& Baert, H. (2010). Theoretical models of participation in adult education: the need for an integrated model. International journal of lifelong education, 1, 45-61. https://doi.org/ 10.1080/02601370903471270.

Bromme, R., Prenzel, M., \& Jäger, M. (2016). Empirische Bildungsforschung und evidenzbasierte Bildungspolitik. Zum Zusammenhang von Wissenschaftskommunikation und Evidenzbasierung in der Bildungsforschung. Zeitschrift für Erziehungswissenschaft, 19(Suppl 1), 129-146.

Buchhaas-Birkholz, D. (2010). Die ,empirische Wende“ in Bildungspolitik und Bildungsforschung. Zum Paradigmenwechsel des BMBF in der Forschungsförderung. DIE Zeitschrift, IV, 30-33.

Bücker, N. (2018). Evangelische Erwachsenenbildung „,neu vermessen“. Präsentation des ersten Bildungsberichts zu evangelischem Bildungshandeln mit Erwachsenen in Hannover. Forum Erwachsenenbildung, 3, 50-51.

Bücker, N., \& Seiverth, A., mit einem Beitrag von P. Schreiner. (2019). Evangelische Erwachsenenbildung: Empirische Befunde und Perspektiven. (Comenius-Institut, Hrsg.). Münster: Waxmann.

Bundesamt für Migration und Flüchtlinge (2017). Bericht zur Integrationskursgeschäftsstatistik für das Jahr 2016. Abfragestand: 03.04.2017. https://www.bamf.de/SharedDocs/Anlagen/DE/Downloads/ Infothek/Statistik/Integration/2016/2016-integrationskursgeschaeftsstatistik-gesamt_bund.html. Zugegriffen: 26. Okt. 2017.

Bundesarbeitskreis ARBEIT UND LEBEN (o. J.) Geschäftsbericht 2014-2015, Wuppertal. https://www. arbeitundleben.de/publikationen/item/geschaeftsbericht-2014-2015. Zugegriffen: 27. Aug. 2018.

Deutscher Bildungsrat (Hrsg.). (1970). Strukturplan für das Bildungswesen. Stuttgart: Klett. 
Deutscher Volkshochschul-Verband (2018). Jahresbericht 2017 (Deutscher Volkshochschul-Verband, Hrsg.) (Nr. 48), Bonn. https://www.dvv-vhs.de/fileadmin/user_upload/3_Der_Verband/Presse/ Jahresberichte/DVV_JB17_Webversion_final.pdf. Zugegriffen: 31. Aug. 2018.

Deutsches Institut für Erwachsenenbildung (Hrsg.). (2014). Trends der Weiterbildung. DIE-Trendanalyse 2014. DIE spezial. Bielefeld: W. Bertelsmann.

Engels, M. (2018). Zwischen Rechenschaftslegung und Wahrheitsfindung. Zeitschrift für Weiterbildungsforschung, 41(1), 27-40. https://doi.org/10.1007/s40955-018-0104-x.

Eurostat \& Europäisches Statistisches System. (2018). Verhaltenskodex für europäische Statistiken für die nationalen statistischen Ämter und Eurostat (statistisches Amt der EU). Luxemburg: Amt für Veröffentlichungen der Europäischen Union. Abgerufen von https://www.destatis.de/DE/Methoden/ Qualitaet/verhaltenskodex.pdf?_blob=publicationFile\&v=3. Zugegriffen: 06. Mai 2019.

Expertenkommission Finanzierung Lebenslanges Lernen (2004). Der Weg in die Zukunft. Schlussbericht. Schriftenreihe der Expertenkommission Finanzierung Lebenslangen Lernens, Bd. 8. Bielefeld: W. Bertelsmann.

Feld, T.C., Schemmann, M., \& Seitter, W. (2016). Weiterbildungsbericht Hessen 2015. Landeskuratorium für Weiterbildung und Lebensbegleitendes Lernen in Zusammenarbeit mit dem Hessischen Kultusministerium (Hrsg.), Wiesbaden. https://kultusministerium.hessen.de/sites/default/files/media/ hkm/weiterbildungsbericht.pdf. Zugegriffen: 30. Aug. 2018.

Fiebig, C. (2016). Gut gerüstet für die Reise in die Zukunft. Landesverband Baden-Württemberg unterstützt die Volkshochschulen mit systematischen Prognosen. dis.kurs, 1, 25-26.

Fleige, M., \& Reichart, E. (2014). Statistik und Programmanalyse als Zugänge zur Angebotsforschung: Erkundungen am Beispiel der kulturellen Bildung in der Volkshochschule. In H. Pätzold, H. von Felden \& S. Schmidt-Lauff (Hrsg.), Programme, Themen und Inhalte der Erwachsenenbildung (S. 68-87). Baltmannsweiler: Schneider Hohengehren.

Gräsel, C. (2011). Was ist empirische Bildungsforschung? In H. Reinders, H. Ditton, C. Gräsel \& B. Gniewosz (Hrsg.), Empirische Bildungsforschung (S. 13-27). Wiesbaden: Springer VS. https://doi.org/10. 1007/978-3-531-93015-2_1.

Gschwend, T. (2006). Ökologische Inferenz. In J. Behnke, T. Gschwend, D. Schindler \& K.-U. Schnapp (Hrsg.), Methoden der Politikwissenschaft: Neuere qualitative und quantitative Analyseverfahren (S. 227-237). Baden-Baden: Nomos.

Herbrechter, D., \& Schemmann, M. (2010). Wandel der Volkshochschulfinanzierung? Eine Analyse der VHS-Statistik. In C. Hof, J. Ludwig \& B. Schäffer (Hrsg.), Erwachsenenbildung im demographischen und sozialen Wandel. Dokumentation der Jahrestagung der Sektion Erwachsenenbildung der Deutschen Gesellschaft für Erziehungswissenschaft, Hochschule für Philosophie München, der Ludwig-Maximilians-Universität München und der Universität der Bundeswehr München, 24. bis 26. September 2009. (S. 171-184). Baltmannsweiler: Schneider-Verlag Hohengehren.

Horn, H., Lux, T., \& Ambos, I. (2018). Weiterbildungsstatistik im Verbund 2016 - Kompakt, Bonn. https:// www.die-bonn.de/doks/2018-weiterbildungsstatistik-02.pdf. Zugegriffen: 10. Dez. 2018.

Huba, H., \& Zierer, W. (2007). Radar für wirtschaftliche Risiken. Das Kennzahlen-Set für Volkshochschulen. dis.kurs, 2, 30-31.

Huntemann, H. (2014). Datenbericht Volkshochschul-Statistik, Bonn. https://www.die-bonn.de/ weiterbildung/statistik/vhs-statistik/Datenbericht_VHS\%20Statistik_20141218.pdf. Zugegriffen: 27. Apr. 2018.

Käpplinger, B. (2017). Die (Un-)Verantwortlichkeit von Verbänden und Trägern für die „Allmenden der Weiterbildung“. Magazin Erwachsenenbildung.at, 11(2017), 06/02-06/09.

Katholische Erwachsenenbildung Deutschland Bundesarbeitsgemeinschaft, K.E. B. (2017). Jahresbericht 2016-2017. https://keb-deutschland.de/wp-content/uploads/2017/07/KEB-Jahresbericht-20162017.pdf. Zugegriffen: 10. Dez. 2018.

Kreisvolkshochschule Groß-Gerau (2017). Jahresbericht 2017, Groß-Gerau. https://www.kvhsgg.de/ fileadmin/redaktion/pdf/Jahresbericht2017neu.pdf. Zugegriffen: 10. Dez. 2018.

Kuper, H., Widany, S., \& Kaufmann, K. (2016). Empfehlungen für eine Statistik zur Bildung Erwachsener in Deutschland. Strategien, Indikatoren und Datengewinnung. In H. Kuper, F. Behringer \& J. Schrader (Hrsg.), Entwicklung von Indikatoren und einer Datengewinnungsstrategie für die Weiterbildungsstatistik in Deutschland (S. 74-106). Bonn: BIBB.

Landesamt für Schulentwicklung/Statistisches Landesamt Baden-Württemberg (2015). Bildung in Baden-Württemberg. Bildungsberichterstattung 2015, Stuttgart. https://www.ls-bw.de/site/pbs-bwnew/get/documents/KULTUS.Dachmandant/KULTUS/Dienststellen/ls-bw/Service/Bildungsbericht erstattung/Bildungsberichte/Bildungsbericht_2015/Bildungsbericht_BW_2015.pdf. Zugegriffen: 10. Dez. 2018. 
Lindemann, W. (2017). VHS-Leiter Freiberger plädiert für zeitgemäßere Programmangebote. Westfälischer Anzeiger vom 17.11.2017. https://www.wa.de/lokales/boenen/vhs-leiter-freiberger-plaediertzeitgemaessere-programmangebote-vhs-zweckverband-bergkamen-boenen-9368924.html. Zugegriffen: 24. Okt. 2018.

Martin, A., \& Muders, S. (2018). Zum Nutzen von Kooperationen für Volkshochschulen. Zeitschrift für Erziehungswissenschaft, 21, 383-405. https://doi.org/10.1007/s11618-017-0779-6.

Martin, A., \& Reichart, E. (2019, im Erscheinen). Zum Einfluss der politischen Bildung an Volkshochschulen auf die Wahlbeteiligung. In J. Schrader, H.P. Blossfeld und A. Ioannidou (Hrsg.). Erträge von Weiterbildung. Zeitschrift für Erziehungswissenschaft - Edition.

Martin, A., \& Schrader, J. (2016). Deutscher Weiterbildungsatlas - Kreise und kreisfreie Städte. Ergebnisbericht, Bonn. https://www.die-bonn.de/doks/2016-weiterbildungsangebot-01.pdf. Zugegriffen: 4. Juli 2016.

Mayert, A. (2017). Wie steht es um Arbeitsbedingungen in Einrichtungen der Evangelischen Erwachsenenbildung? forum erwachsenenbildung, 1(17), 32-36.

Mikschl, J. (2018). Vernachlässigt: Volkshochschule. Erziehung \& Wissenschaft. Zeitschrift der Bildungsgewerkschaft GEW, 07-08(18), 16-17.

Pehl, K. (2007). Strategische Nutzung statistischer Weiterbildungsdaten. Leitfaden für Einrichtungen und Verbände. Bielefeld: W. Bertelsmann.

Pehl, K. (2013). Sichtbarkeit von Weiterbildungseinrichtungen - Statistik als Abbildung von Weiterbildungsleistungen. In T. Feld, S. Kraft, S. May \& W. Seitter (Hrsg.), Engagierte Beweglichkeit. Weiterbildung in öffentlicher Verantwortung (S. 185-200). Wiesbaden: Springer.

Reichart, E. (2006). Verbund Weiterbildungsstatistik liefert regelmäßig Institutionendaten zur allgemeinen Weiterbildung, Bonn. https://www.die-bonn.de/esprid/dokumente/doc-2006/reichart06_02.pd. Zugegriffen: 23. Aug. 2018.

Reichart, E. (2012). Kennzahlen über Weiterbildungseinrichtungen. Welche es bisher gibt, wo sie Anwendung finden und wo noch Lücken bestehen. Der pädagogische Blick, 20(3), 143-163.

Reichart, E. (2018a). Berufliche Weiterbildung an Volkshochschulen. In Bundesinstitut für Berufsbildung (Hrsg.), Datenreport zum Berufsbildungsbericht 2018. Informationen und Analysen zur Entwicklung der beruflichen Bildung (1. Aufl. S. 374-377). Bonn: Barbara Budrich. Kap. B 2.2.1, https://nbnresolving.de/urn:nbn:de:0035-0729-4. Zugegriffen: 10.12.2018.

Reichart, E. (2018b). 55 Jahre Erwachsenenbildung im Spiegel der Statistik - die deutschen Volkshochschulen und ihr Angebot seit 1962. Bildung und Erziehung, 71(2), 186-200.

Reichart, E., \& Rattinger, J. (2017). Die statistische Entwicklung des Programmbereichs Arbeit - Beruf an Volkshochschulen seit den 1990er Jahren. Tiefenanalysen und Interpretationen aus der Perspektive der Praxis. Hessische Blätter für Volksbildung, 67, 246-256. https://doi.org/10.3278/HBV1703W246.

Reichart, E., Lux, T. \& Huntemann, H. (2018). Volkshochschul-Statistik. 56. Folge, Arbeitsjahr 2017. DIE Survey. Abgerufen von https://www.die-bonn.de/doks/2018-volkshochschule-01.pdf. Zugegriffen: 06. Mai 2019

Schneider, C., Klostermann, T., \& Reichart, E. (2014). Datengrundlage und Datenzugänge in einem kommunalen Bildungsmonitoring. In K. Drossel, R. Strietholt \& W. Bos (Hrsg.), Empirische Bildungsforschung und evidenzbasierte Reformen im Bildungswesen (S. 179-196). New York, Münster: Waxmann.

Schön, T. (2014). Kurzinformation. Große Revision der DIE-Anbieter-/Angebotsstatistiken. Kontext und Ziele des Projekts, Bonn. https://www.die-bonn.de/doks/2014-statistik-01.pdf. Zugegriffen: 10. Dez. 2018.

Schrader, J. (2010a). Reproduktionskontexte der Weiterbildung. Zeitschrift für Pädagogik, 56(2), $267-284$.

Schrader, J. (2010b). Pluralismus. In R. Arnold, S. Nolda \& E. Nuissl (Hrsg.), Wörterbuch Erwachsenenbildung (2. Aufl. S. 239-240). Bad Heilbrunn: Klinkhardt.

Schuldt, H.-J. (Hrsg.). (1998). Mit Kennzahlen arbeiten. Beiträge zur Kennzahlendiskussion bei Weiterbildungseinrichtungen, Bonn: DIE, texte.online. https://www.die-bonn.de/esprid/dokumente/doc-1998/ schuldt98_01.pdf. Zugegriffen: 10. Dez. 2018.

Seitter, W. (2013). Kennziffern und innersprachliche Mehrsprachigkeit. Zur kommunikativen Arbeit mit Zahlen in Weiterbildungseinrichtungen. In T. Feld, S. Kraft, S. May \& W. Seitter (Hrsg.), Engagierte Verantwortung. Weiterbildung in öffentlicher Verantwortung (S. 177-184). Wiesbaden: Springer.

Senatsverwaltung für Bildung, Jugend und Familie (2018). VHS-Statistik 2017, Berlin. https://www.berlin. de/sen/bildung/lebenslanges-lernen/vhs/fachinfo/. Zugegriffen: 3. Dez. 2018.

Statistisches Bundesamt (2017). Weiterbildung - 2017, Wiesbaden. https://www.destatis.de/DE/ Publikationen/Thematisch/BildungForschungKultur/Weiterbildung/BeruflicheWeiterbildung 
5215001177004.pdf;jsessionid=AF5FF4B6D7E8FA85DFEE3099602AFF99.InternetLive1? blob=publicationFile. Zugegriffen: 16. Juli 2018.

Terhart, E. (2001). Bildungsforschung, Bildungsadministration, Bildungswirklichkeit. Eine systematische Annäherung. In K.J. Tillmann \& W. Vollstädt (Hrsg.), Politikberatung durch Bildungsforschung. Schule und Gesellschaft (Bd. 27, S. 17-32). Wiesbaden: Springer.

Thüringer Volkshochschulverband e. V. (2017). Jahresbericht 2016, Jena. https://www.vhs-th.de/fileadmin/ redaktion/Der_Verband/Jahresbericht_2016_Web.pdf. Zugegriffen: 31. Aug. 2018.

Weber, M. (2017). Die VHS-Statistikreform und das Erfassen von berufsbezogenen Kursen im Kursprogramm der Volkshochschulen. Hessische Blätter für Volksbildung, 3, 281-284.

Wittenbrink, L., \& Frick, J. (2018). Deutscher Weiterbildungsatlas. Teilnahme und Angebot in Kreisen und kreisfreien Städten, Gütersloh. https://www.bertelsmann-stiftung.de/fileadmin/files/user_upload/ Deutscher_Weiterbildungsatlas_Staedte_Laender_2018.pdf. Zugegriffen: 10. Dez. 2018.

Wolter, A., Widany, S., \& Dollhausen, K. (2019). Monitoring wissenschaftlicher Weiterbildung. In W. Jütte \& M. Rohs (Hrsg.), Handbuch wissenschaftliche Weiterbildung. Wiesbaden: Springer VS. Manuskript in Vorbereitung.

Wuppertaler Kreis, e. V. (2017). Trends in der Weiterbildung. Verbandsumfrage 2017, Köln. http://www. wkr-ev.de/trends17/wktrends2017.pdf. Zugegriffen: 23. Aug. 2018.

ZDF, Zweites Deutsches Fernsehen (2017). Deutsch als Fremdsprache beliebt. ZDF, heute Nachrichten vom 05.12.2017. https://www.zdf.de/nachrichten/heute/an-den-volkshochschulen-deutsch-alsfremdsprache-beliebt-100.html. Zugegriffen: 24. Okt. 2018.

Publisher's Note Springer Nature remains neutral with regard to jurisdictional claims in published maps and institutional affiliations. 